\title{
Efficacy of "Oil Pulling" on Gingival Health and Biofilm Accumulation in a Group of Young Adults: A Pilot Study
}

\author{
${ }^{1}$ Sara Rayner, ${ }^{2}$ Trislyn Herrick, ${ }^{3}$ Christina Chiesa, ${ }^{4}$ Jay Hoover, ${ }^{5}$ Chandima Karunanayake
}

\begin{abstract}
Aims: The aim of this pilot study was to assess changes in gingival bleeding and biofilm accumulation in young adults undergoing oil-pulling treatment together with standardized oral hygiene practices, compared to a control group of patients that did not undergo oil pulling but maintained the standard oral hygiene practices outlined in the study.
\end{abstract}

Materials and methods: The experiment was designed as a randomized, two-way, single-blind, crossover with a 2-month washout period between two, 10-day experimental periods. A total of 28 , randomly selected dental students who met the inclusion criteria were assessed for gingival bleeding and biofilm on six teeth before and after rinsing with sesame oil for 10 minutes per day, for a period of 10 days. The mean difference between the test and control group's reduction in scores was analyzed using the paired t-test. The level of significance was set at $\alpha($ alpha $)=0.05$.

Results: There were no statistically significant differences on plaque biofilm accumulation and bleeding when compared with the control group. There was a higher reduction in gingival bleeding scores (albeit not statistically significant) among those who used sesame oil compared to those who did not.

Conclusion: Based on the current study, it appears that oil pulling for 10 minutes and for a period of 10 days using sesame oil has no significant benefit over brushing and flossing alone, on a sample of 28 dental students.

Keywords: Biofilm, Experimental dental science, Gingivitis, Oil pulling, Sesame oil.

How to cite this article: Rayner S, Herrick T, Chiesa C, Hoover J, Karunanayake C. Efficacy of "Oil Pulling" on Gingival Health and Biofilm Accumulation in a Group of Young Adults: A Pilot Study. Int J Experiment Dent Sci 2016;5(2):113-117.

Source of support: Nil

Conflict of interest: None

\footnotetext{
${ }^{1-3}$ Dental Student, ${ }^{4}$ Professor, ${ }^{5}$ Professional Research Associate

${ }^{1-4}$ Department of Periodontics, College of Dentistry, Saskatoon Saskatchewan, Canada

${ }^{5}$ Canadian Centre for Health and Safety in Agriculture, University of Saskatchewan, Saskatoon, Saskatchewan, Canada
}

Corresponding Author: Jay Hoover, Professor, Department of Periodontics, College of Dentistry, Saskatoon, Saskatchewan Canada, Phone: +3069665110, e-mail: j.hoover@usask.ca

\section{INTRODUCTION}

Complementary and alternative medicines are attaining acceptance over allopathic medicine in Western societies for varying reasons, and one of the most popular modalities of alternative therapy is the practice of ayurvedic medicine. ${ }^{1}$ Ayurveda, a traditional holistic form of medicine practiced in the Indian subcontinent for over hundreds of years, utilizes plant-based active alkaloids as pharmaceutical remedies to treat a variety of topical and systemic illnesses. One such practice is the use of plant-based edible oils, termed "oil pulling," to improve oral health. Historically, it is thought that the practice of oil pulling strengthens teeth, gingival tissues, and jaws; prevents tooth decay, oral malodor, periodontal diseases, dryness of the throat, and cracked lips; and is also believed to have the capacity to treat systemic diseases ranging from headaches and migraines to diabetes and asthma. ${ }^{2,3}$ However, the precise physiological mechanism of action has not been well researched or understood. ${ }^{4}$ The term "oil pulling" stems from the holistic belief that swishing the oil for a period of time within the oral cavity will activate enzymes and draw or "pull" toxins from the teeth, saliva, and blood. Since the oral mucosa is not a semipermeable membrane, it is unlikely that toxins can be drawn out of the blood.

Many edible oils, such as sesame seed, sunflower seed, or coconut oil, can be used for the oil-pulling process. Sesame oil is extracted from seeds of the plant Sesamum indicum by pressing the seeds. ${ }^{5}$ This oil in particular contains the antioxidants sesamin, sesamolin, and sesaminol, which are thought to contribute to various positive therapeutic outcomes. Bacterial infections of the oral cavity, such as chronic gingivitis produce a host immune response that includes the release of a high amount of free radicals, culminating in heightened oxidative damage to gingival tissues, periodontal ligament, and alveolar bone. ${ }^{6}$ The antioxidative properties of sesame oil create an equilibrium with free radical production to decrease oxidative stress on the oral tissues, which, in turn, may help lessen the potential of pathological changes to the teeth, gingiva, and other oral tissues. ${ }^{6}$ Sesamin, sesamolin, and sesaminol lignans found in sesame oil have been shown to also enhance the antioxidative properties 
of dietary vitamin E. ${ }^{4}$ A study by Asokan et $\mathrm{al}^{5}$ showed that saponification and emulsification occur while oil pulling with sesame seed oil, which may enhance the cleansing action of the oil. The same process may perhaps contribute to the removal of stains reported by some users. The constant swishing and pulling of the oil in the oral cavity and between the teeth is also assumed to be a factor in the mechanical disruption of bacterial accumulation and biofilm formation, leading to better oral hygiene and decreases in carious lesions and periodontal inflammation. ${ }^{5}$ Thaweboon et $\mathrm{al}^{7}$ comparing the use of different edible oils on biofilm models formed by Streptococcus mutans KPSK2, Lactobacillus casei ATCC 6363, and Candida albicans ATCC 13803 on salivary-coated microtiter plates reported that sesame oil had an antibacterial effect against $S$. mutans (1 log reduction). Similar results were obtained in a group of 20 adolescents after oil pulling with sesame oil and compared to rinsing with chlorhexidine. ${ }^{4}$ The authors reported a reduction in $S$. mutans count in the plaque and saliva samples in the oil group, but the difference was not significant over the entire time period of the experiment when compared to those who rinsed with chlorhexidine. Recently Sood et $\mathrm{al}^{8}$ reported that oil pulling with sesame oil was equally efficacious as chlorhexidine in reducing oral malodor and microbes causing it. Benefits of rinsing the oral cavity with oil as opposed to standard mouthwashes include decreased staining, aftertaste, and allergies. ${ }^{4}$ A disadvantage to oil pulling is that it is time consuming and there is skepticism as to whether or not it is more effective than simply brushing for 2 minutes twice a day. If indeed oil pulling consistently demonstrates anti-inflammatory and caries reduction potential and considering that these oils are readily available at a low cost, it may be a useful preventative strategy for those who cannot afford regular professional dental care, non-ambulatory persons, or those physically challenged. This practice may improve the oral health of people in developing nations who face barriers to regular dental care, live in refugee camps, or are marginalized members of society. ${ }^{4}$ Recently, pulling oil for therapeutic purposes is becoming a popular practice in Western society. There is, however, at the present time, only a relatively small number of published data on the effect of oil pulling on gingival inflammation, caries reduction, and biofilm accumulation.

The aim of this pilot study was to assess changes in gingival bleeding and biofilm accumulation in young adults undergoing oil-pulling treatment together with standardized oral hygiene practices, compared to a control group of patients that did not undergo oil pulling but maintained the standard oral hygiene practices outlined in the study. In addition, a qualitative visual assessment of salivary bacterial colony growth and the effect of tooth whitening before and after oil pulling was done.

\section{MATERIALS AND METHODS}

A sample of 30 participants, aged 20 to 26 years were randomly selected from 1st- and 2nd-year students currently enrolled at the College of Dentistry, University of Saskatchewan, Saskatoon, Canada. Inclusion criteria were as follows: Dentate, medically healthy, no orthodontic bands, and an anticipated ability to attend all four scheduled visits. Exclusion criteria included: A significant medical condition (including, but not limited to, diabetes mellitus, rheumatic heart disease, or clinically significant heart murmur), pregnancy, and a recent history of, or ongoing, antibiotic therapy. All potential subjects were screened prior to the exam and selected as per the inclusion/exclusion criteria. The study was conducted in full accordance with ethical principles and with the approval of the University of Saskatchewan, Biomedical Research Ethics Board. Participants signed an informed consent form and were free to withdraw from the trial at any time.

\section{Study Protocol}

The experiment was designed as a randomized, two-way single-blind, crossover with a 2-month washout period between two, 10-day experimental periods. The subjects were divided into two groups of 15 with group A being the control and group B the test group.

The clinical examinations were conducted at the dental clinic, College of Dentistry, University of Saskatchewan, by one of the investigators (SR) who remained blind throughout the study period. This examiner was trained and calibrated, and an intra-examiner variability test was performed to ensure the accuracy of the recordings. At baseline, the outcome variables were the Plaque Index ${ }^{9}$ and the presence or absence of gingival bleeding on gentle probing of the sulcus. Each of the outcome variables was measured on the mesial, distal, lingual, and buccal surfaces of six representative teeth: $16,21,24,36,41$, and 44 . Tooth shade was determined on the upper central incisor using the VITA easyshade ${ }^{\circledR}$ Compact digital shade measuring device. The shade guide (B1, A1, B2, D2, A2, C1, C2, D4, A3, D3, B3, A3.5, $\mathrm{B} 4, \mathrm{C} 3, \mathrm{~A} 4$, and $\mathrm{C} 4$ ) was replaced by numeric values 1 to 16 for analysis, with the lightest shade assigned the smallest value. Unstimulated saliva samples were obtained from all participants and tested for total bacterial colony growth on Blood Agar and MacConkey Agar Plates, which were incubated for 24 hours. Photos were taken for a qualitative visual assessment of bacterial colony growth. At the conclusion of the baseline measurements, the first group (I) was asked to maintain standardized oral health practices 
for the duration of the experiment in which they were required to brush their teeth for 2 minutes, twice per day and flossing once daily using the provided dentifrice. Group II (test group) participants were requested to swish a tablespoon of the provided unrefined, expeller pressed sesame seed oil (Spectrum Naturals ${ }^{\circledR}$ ) in the mouth for 10 minutes once per day in the morning on an empty stomach and then dispose of the oil preferably in a paper towel. Oil pulling was to be followed by tooth brushing with the provided dentifrice and flossing as above. Written instructions on the oilpulling technique was also given to the participants. After a 10-day trial period, subjects in both groups were reassessed as per baseline (phase 1). After a washout period of 2 months, the experiment was then repeated, switching participants in the test and control groups (phase 2). Two subjects did not participate in the second phase of the study. Therefore, those two subjects were excluded from the analysis, and there were 28 subjects in total, in each group. Plaque biofilm, bleeding scores, and tooth shade were again recorded at baseline and after 10 days. A short post-experimental questionnaire comprising of two questions, "Please comment on any discernable changes you noticed in your oral health during or after the oil pulling process (such as changes in breath, cleanliness, whiteness, bleeding or gingival health/ color)?" and "Would you consider adding oil pulling into your daily oral hygiene regimen? Why or why not?," was administered to the participants at the end of the trial.

\section{Statistical Analysis}

For phase 1 of the study, the reduction from the baseline in the plaque and bleeding scores were calculated taking the difference between the scores at baseline and after the 10-day trail period. Similarly, for phase 2 of the study, after the washout period, the reduction from the baseline in the plaque and gingivitis scores were calculated taking the difference between the scores at baseline and after the 10-day trial period. The observed data, test scores (those who used sesame oil), and control scores (those who did not use sesame oil) were from the same subject, and it was assumed that the sample was drawn from a population with a normal distribution. Hence, the mean difference between test and control group's reduction in scores was analyzed using the paired t-test. The level of significance was set at $\alpha$ (alpha) $=0.05$.

\section{RESULTS}

The effects of rinsing the mouth (oil pulling) with sesame oil for a period of 10 minutes for 10 days on plaque biofilm and bleeding on gentle probing of the sulcus are shown in Table 1 . There was no statistically significant difference in the overall reduction from baseline in relation to the mean plaque scores and the mean gingivitis scores for all surfaces when compared with the control group. However, there was a higher reduction in gingival bleeding scores (albeit not statistically significant) among those who used sesame oil compared to those who did not.

Tables 2 and 3 show the effect of oil pulling on the individual surfaces (buccal, mesial, lingual, and distal) on plaque inhibition and gingivitis. There were no statistically significant differences on any of the four surfaces examined when compared with the control group. The results of the current study also did not

Table 1: Overall reduction from baseline in the mean plaque scores and the mean gingivitis scores:

Buccal, mesial, lingual, distal surfaces

\begin{tabular}{lcccrc}
\hline Tooth surface & Test $\pm S D$ & Control $\pm S D$ & Difference $\pm S D$ & Paired $t$-value & $p$-value \\
\hline Plaque & $0.07 \pm 0.09$ & $0.05 \pm 0.09$ & $0.02 \pm 0.15$ & 0.663 & 0.513 \\
Gingivitis & $-0.13 \pm 0.16$ & $-0.05 \pm 0.23$ & $-0.08 \pm 0.25$ & -1.540 & 0.135 \\
\hline
\end{tabular}

Table 2: Overall reduction from baseline in the mean plaque scores and the mean gingivitis: Buccal, mesial, lingual, distal surfaces

\begin{tabular}{llllrl}
\hline Tooth surface & Test $\pm S D$ & Control $\pm S D$ & Difference $\pm S D$ & Paired $t$-value & $p$-value \\
\hline Buccal & $0.10 \pm 0.14$ & $0.08 \pm 0.16$ & $0.02 \pm 0.18$ & 0.515 & 0.611 \\
Mesial & $0.01 \pm 0.10$ & $0.02 \pm 0.10$ & $-0.01 \pm 0.18$ & -0.176 & 0.861 \\
Lingual & $0.08 \pm 0.18$ & $0.07 \pm 0.19$ & $0.01 \pm 0.31$ & 0.103 & 0.919 \\
Distal & $0.08 \pm 0.17$ & $0.02 \pm 0.18$ & $0.06 \pm 0.27$ & 1.170 & 0.252 \\
\hline
\end{tabular}

Table 3: Reduction from baseline in the mean gingivitis scores: Buccal, mesial, lingual, distal surfaces

\begin{tabular}{lccccc}
\hline Tooth surface & Test $\pm S D$ & Control $\pm S D$ & Difference $\pm S D$ & Paired $t$-value & $p$-value \\
\hline Buccal & $-0.11 \pm 0.26$ & $-0.04 \pm 0.24$ & $-0.07 \pm 0.33$ & -1.151 & 0.260 \\
Mesial & $-0.13 \pm 0.32$ & $-0.05 \pm 0.36$ & $-0.08 \pm 0.43$ & -0.944 & 0.354 \\
Lingual & $-0.17 \pm 0.34$ & $-0.12 \pm 0.27$ & $-0.05 \pm 0.48$ & -0.593 & 0.558 \\
Distal & $-0.09 \pm 0.25$ & $0.01 \pm 0.37$ & $-0.10 \pm 0.38$ & -1.315 & 0.200 \\
\hline
\end{tabular}


Table 4: Comparison of color shade codes between groups (treatment and control) for tooth \#11

\begin{tabular}{llllll}
\hline Tooth surface & Test $\pm S D$ & Control $\pm S D$ & Difference $\pm S D$ & Paired $t$-value & $p$-value \\
\hline$\# 11$ & $0.36 \pm 1.39$ & $0.18 \pm 0.61$ & $0.18 \pm 1.68$ & 0.563 & 0.578 \\
\hline
\end{tabular}

indicate any statistically significant changes in tooth shade between those who rinsed with oil compared to subjects who did not (Table 4). In addition, inoculated saliva samples from the test subjects showed no visual difference in gram positive bacteria colonies when compared with the controls.

The short post-experiment questionnaire elicited the following responses. With regard to question 1, most respondents felt that it improved parameters of oral health, such as better breath and an overall cleaner-feeling mouth, while some experienced increased gingival sensitivity and bleeding on flossing. With regard to question 2, a few subjects thought the time commitment was excessive and inconvenient, while others indicated that they would continue oil pulling based on feelings of relaxation and increased cleanliness.

\section{DISCUSSION}

Despite a paucity of published research attesting not only its efficacy within the oral cavity but also its purported effect on selected systemic diseases, oil pulling appears to be gaining popularity as per an internet search in North America. This may probably be due to an increase in interest in naturopathy, ayurveda, and other forms of alternate medicine and the health benefits of edible vegetable oils, such as coconut oil. The few studies investigating the effect of oil pulling have been mostly carried out in the Indian subcontinent, ${ }^{1-8,10}$ and the present study is probably the first in North America, to the best of the authors knowledge based on a search in PubMed, Scopus, and other databases, investigating the effect of rinsing the oral cavity with sesame oil on gingival bleeding and plaque biofilm.

The results of this study did not indicate a statistically significant difference in the reduction of plaque accumulation or gingival bleeding between the test (oil pulling) and control groups. Conversely, Asokan et $\mathrm{al}^{11}$ reported that after oil pulling with sesame oil, a reduction in the plaque index, modified gingival scores, and total colony count of aerobic microorganisms in the plaque were observed in a group of 20, age-matched adolescent boys attending a school in India. As there is a scarcity of published reports on the effect of oil pulling on plaque and gingival bleeding, it is not possible to compare further the results of the present study.

Hannig et $\mathrm{al}^{10}$ investigated, in vitro, the effect of selected edible oils, such as safflower, olive, and linseed on the early colonization of enamel surfaces. They reported that rinsing with edible oils had no significant influence on the initial pattern and the quantity of bacterial colonization on enamel over an 8-hour period and hence could not recommend rinsing with oils to reduce oral biofilm formation. Similarly, the results of the current clinical study comparing inoculated saliva samples before and after oil pulling showed no visual difference in Gram positive bacterial colonies. However, to accurately determine the difference in the number of colony-forming units, a quantitative culture using a selective or differential media to target specific pathogen growth would need to be performed. On the contrary, some in vitro studies have demonstrated that a few types of edible oils have antibacterial activity. ${ }^{7}$ Thaweboon et al ${ }^{7}$ in 2011 reported that sesame oil had antibacterial activity against $S$. mutans, whereas sunflower oil had antifungal activity against $C$. albicans. However, $L$. casei was resistant to all tested oils. In 2012, a group from the Athlone Institute of Technology, Ireland, reported that coconut oil, partially pre-digested with enzymes, inhibited the growth of most strains of Streptococcus bacteria including S. mutans. ${ }^{12}$ There is no published data available to date, on the effect of edible oils on periodontal pathogens.

Although an Internet search revealed some anecdotal accounts claiming that oil pulling tends to brighten teeth, possibly due to its emulsifying effect, ${ }^{5}$ the results of the current study did not indicate any statistically significant changes in tooth shade before and after rinsing with oil. Dental students were utilized as subjects as they were present on site and were able to attend all scheduled examinations. It was also presumed that the students would be more compliant to following instructions specially pertaining to oil pulling than the population at large. However, dental students in general are inclined to have better oral hygiene and supposedly more motivated than the general population, and consequently any changes in gingival bleeding and plaque inhibition before and after therapy may be assumed to be slight. In order to minimize this limitation, the sample was randomly drawn from 1st and 2nd-year students who tend to have a poorer oral hygiene than their senior counterparts. ${ }^{13}$

Limitations of the present study also included the comparatively small sample size and possibly an issue with compliance regarding the duration of oil pulling as subjects were issued a bottle of oil and instructed to rinse at home and were, therefore, unsupervised. The relatively short follow-up period (10 days) may also be considered a limitation. 


\section{CONCLUSION}

Based on the current study, it appears that oil pulling for 10 minutes and for a period of 10 days using sesame oil has no significant benefit over brushing and flossing alone, in a sample of 28 dental students. However, considering some of the limitations of this pilot study and based on empirical information and its traditional use over a long time period, further basic research concerning the mechanism of action of oil pulling and clinical research involving larger, diverse population groups with a longer follow-up period must be conducted before any decisions could be made concerning its role as a therapeutic agent for chronic gingivitis.

\section{ACKNOWLEDGMENT}

Authors wish to thank all the subjects who willingly participated in the study and Dr Blondeau, Department of Pathology and Laboratory Medicine, University of Saskatchewan, for his expert guidance.

\section{REFERENCES}

1. Hebbar A, Keluskar V, Shetti V. Oil pulling - unraveling the path to mystic cure. J Int Oral Health 2010 Dec;2(4):11-13.

2. Amruthesh S. Dentistry and ayurveda - IV: classification and management of common oral diseases. Indian J Dent Res 2008 Jan-Mar;19(1):52-61.

3. Mathew RA, Sankari M. Oil pulling and its role in oral and systemic diseases - a review article. Int J Pharm Tech Res 2014 Apr-Jun;6(2):701-703.
4. Asokan S, Rathan J, Muthu MS, Rathna PV, Emmadi P, Raghuraman R, Chamunddeswari R. Effect of oil pulling on Streptococcus mutans count in plaque and saliva using Dentocult SM Strip mutans test: a randomized, controlled, triple-blind study. J Indian Soc Pedod Prev Dent 2008 Mar;26(1):12-17.

5. Asokan S, Rathinasamy TK, Inbamani N, Menon T, Senthil Kumar S, Emmadi P, Raghuraman R. Mechanism of oil-pulling therapy - in vitro study. Indian J Dent Res 2011 Jan-Feb;22(1): 34-37.

6. Pendyala G, Thomas B, Kumari S. The challenge of antioxidants to free radicals in periodontitis. J Indian Soc Periodontol 2008 Sep-Dec;12(3):79-83.

7. Thaweboon S, Nakaparksin J, Thaweboon B. Effect of oilpulling on oral microorganisms in biofilm models. Asia J Public Health 2011 May-Aug;2(2):62-66.

8. Sood P, Devi A, Narang R, Swathi V, Makkar D. Comparative efficacy of oil pulling and chlorhexidine on oral malodor: a randomized controlled trial. J Clin Diagn Res 2014 Nov;8(11): ZC18-ZC21.

9. Löe H. The Gingival Index, the Plaque Index and the Retention Index. J Periodontol 1967 Nov-Dec;38(6):610-616.

10. Hannig C, Kirsch J, Al-Ahmad A, Kensche A, Hannig M, Kümmerer K. Do edible oils reduce bacterial colonization of enamel in situ? Clin Oral Investig 2013 Mar;17(2):649-658.

11. Asokan S, Emmadi P, Chamundeswari R. Effect of oil pulling on plaque induced gingivitis: a randomized, controlled, triple-blind study. Indian J Dent Res 2009;20(1):47-51.

12. AIT. AIT researchers show coconut oil could combat tooth decay. Westmeath, Ireland. Athlone Institute of Technology; 2012 Sep. Available from: http://www.ait.ie/aboutaitandathlone/newsevents/pressreleases/2012pressreleases/title16107-en.html.

13. Lang NP, Gumming BR, Löe HA. Oral hygiene and gingival health in Danish dental students and faculty. Community Dent Oral Epidemiol 1977 Oct;5(5):237-242. 